studies have assessed sex differences in outcomes after endovascular stroke thrombectomy of large vessel occlusion (LVO), and none have explored the relationship in a robust realworld registry.

Methods Data from the NeuroVascular Quality InitiativeQuality Outcomes Database (NVQI-QOD) registry was analyzed and compared for sex differences in stroke thrombectomy outcomes in 3261 patients from 23 US centers (17 states) between January 2015 to March 2020. Welch's twosided t-tests, Chi-square tests, and multivariate regression models were used to assess sex differences for 11 outcome variables: final thrombolysis in cerebral infarction (TICI) grade $(\mathrm{n}=3182), 24$ hour NIH stroke score (NIHSS) $(n=2842)$, post-procedure length of stay $(n=3257)$, ICU days $(\mathrm{n}=2787)$, in-hospital mortality $(\mathrm{n}=3259)$, discharge status $(n=3252)$, discharge NIHSS $(n=2426)$, discharge modified Rankin score (mRS) $(n=996)$, days to follow-up $(n=1784)$, 90 day readmission rate $(n=416)$, and 90 day mRS $(n=1184)$. Models controlled for demographics, comorbidities, intravenous tPA thrombolysis, and pre-stroke functional measures.

Results Among the 3261 patients that underwent stroke thrombectomy, $49.4 \%$ were female $(n=1622)$ and $50.6 \%$ were male $(n=1639)$. T-tests and Chi-square tests revealed significant differences between females and males for postprocedure length of stay (8.1 versus 9.1; $\mathrm{p}=0.019), 24$ hour NIHSS (11.1 versus $10.0 ; \mathrm{p}=0.001)$, discharge status $(30.8 \%$ home versus $34.5 \%$ home; $\mathrm{p}<0.001)$, days to follow-up (110 versus $126 ; \mathrm{p}=0.008)$, and in-hospital mortality $(25.3 \%$ versus $19.9 \% ; \mathrm{p}<0.001)$. There were no significant sex differences for discharge mRS without mortality (Female: $27.0 \%$ favorable outcome, Male: $26.7 \%$; $\mathrm{p}=0.95$ ) or for 90 day mRS without mortality (Female: $52.8 \%$ favorable outcome, Male: $49.5 \%$; $\mathrm{p}=0.60)$. Multivariate regression models revealed that females had significantly higher 24 hour NIHSS $(p=0.004)$, fewer days to follow-up $(p=0.033)$, and higher rate of in-hospital mortality $(p=0.036)$. The interaction term of African American $x$ Male and Hispanic x Male were significant for post-procedure length of stay and ICU days, respectively $(p=0.026$ and $\mathrm{p}=0.022$ ), indicating a differential effect of race on the outcome variable within males.

Conclusion Evidence from the NVQI-QOD registry suggests that there are differences between males and females in outcomes after acute stroke thrombectomy, with females showing consistently worse outcomes for short-term functional status, morbidity, and early mortality. Finally, there appears to be a differential effect of race on certain outcome variables within males.

Disclosures V. Thirunavu: None. R. Abdalla: None. D. Cantrell: None. M. Hurley: None. A. Shaibani: None. B. Jahromi: None. M. Potts: None. S. Ansari: None. O. NVQIQOD Registry: None.

\section{LB-005 UNRUPTURED ARTERIOVENOUS MALFORMATION INTERVENTION RATE IS INVERSELY CORRELATED WITH RUPTURED AVM DISCHARGE INCIDENCE}

'D McCarthy*, E Luther' ${ }^{2}$, B Gross ${ }^{1}$, R Starke ${ }^{3}$. 'University of Pittsburgh Medical Center, Pittsburgh, PA; ${ }^{2}$ University of Miami, Miami, FL; ${ }^{3}$ University of Miami Miller School of Medicine, Miami, FL
Introduction The 2013 ARUBA trial concluded that medical management alone is superior to medical management with interventional therapy for patients with unruptured brain arteriovenous malformations.

Objective To analyze AVM treatment and epidemiologic response to the ARUBA trial.

Methods National Inpatient Sample was reviewed from 2003 to 2017, ruptured and unruptured AVMs were identified (rAVM, uAVM). Periods before and after ARUBA defined as 2010-2013 and 2014-2017. Multivariable logistic regression identified correlates to intervention likelihood. Yearly intervention rate (YIR, \#interventions/\#AVM discharges) and yearly rAVM incidence (YRR, \#rAVM/\#AVM discharges) were calculated. Segmented regression (SR) identified significant changepoints and fitted segmented linear models for YIR and YRR. Average annual percent change (AAPC) was estimated as the weighted sum of segmented model slopes. Pearson coefficients (PC) examined correlation between YIR and YRR. RStudio performed statistics.

Results 90,296 AVM discharges were identified from 20102017. AVM discharges were seen at smaller, non-academic hospitals following ARUBA $(\mathrm{p}<0.0001)$. SR identified a significant change in YIR during 2014. Figure 2 shows a decrease in interventions for uAVMs $(28.1 \%$ vs. $22.3 \%$, $\mathrm{p}<0.0001$ ) and no change in rAVM interventions following ARUBA $(p=0.22)$. uAVM discharges following ARUBA had a less likelihood of intervention than those before (OR 0.857 , $\mathrm{p}=0.02$ ); however, intervention likelihood for uAVMs with associated aneurysms were unaffected by ARUBA. Since we unexpectedly observed a higher rAVM discharge incidence following ARUBA $(18.6 \%$ vs $14.7 \%, \mathrm{p}<0.0001)$, the analysis was expanded to 2003 (154,297 discharges). Overall, YRRs have increased (AAPC +0.49\%, $\mathrm{p}=0.0001$ ) and YIRs have decreased (AAPC -1.17\%, $p=0.0001$; figure $1 \mathrm{~A}$ ). The

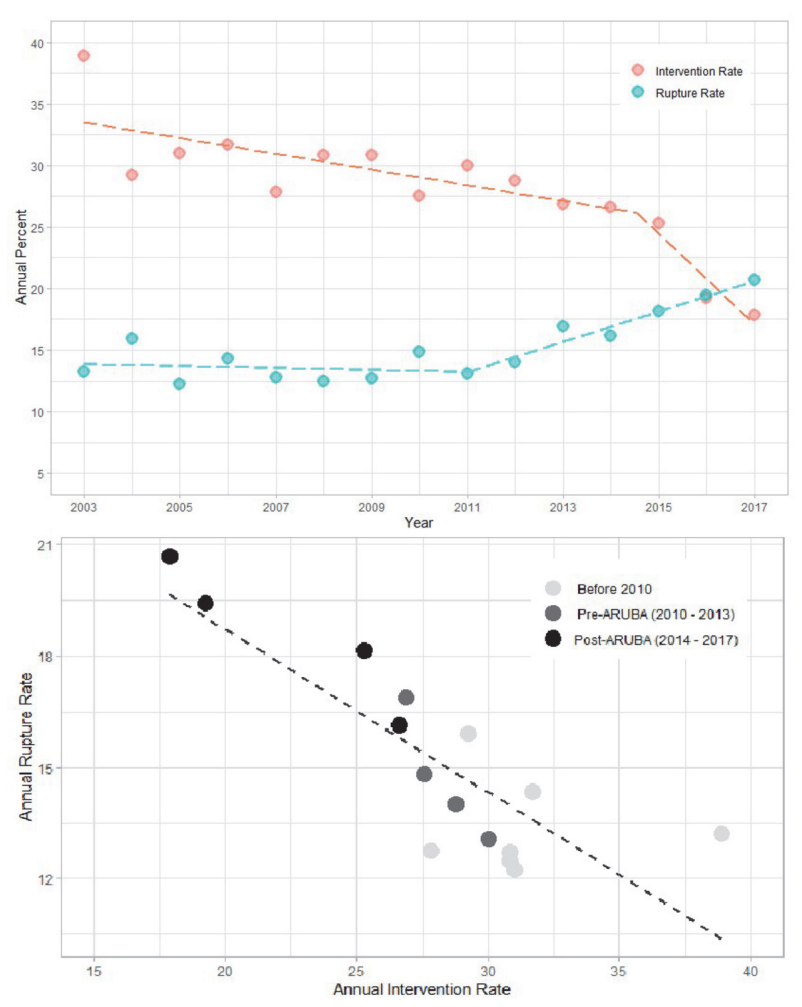

Abstract LB-005 Figure 1 
segmented AAPC for YIR was $-0.63 \%(p=0$. 0.0048) before 2014 and $-3.71 \%(p=0.0 .0596)$ after 2014. Segmented YRR AAPC was $-0.07 \% \quad(p=0.67)$ before 2011 and $+1.24 \%$ $(\mathrm{p}=0.0006)$ after 2011. An 8-year latency between YIR decrease and significant YRR change was observed. In 2017, the YRR (20.6\%) surpassed the YIR (19.7\%) for the first time. YIRs had a strong inverse correlation with YRRs $(\mathrm{PC}=-$ $0.82, \mathrm{p}=0.0002$; figure $1 \mathrm{~B}$ ).

Conclusion Following ARUBA, there has been a stark decrease in interventions for uAVMs. Nationwide rAVM discharge incidence is inversely correlated with uAVM intervention rate. Less uAVM interventions may lead to a substantial increase in AVM rupture incidence.

Disclosures D. McCarthy: None. E. Luther: None. B. Gross: None. R. Starke: None.

\section{LB-006 INCREASED INTRACRANIAL AND SYSTEMIC VCAM1 RELATES TO HYPERTENSION AND REDUCED PERCENT CHANGE IN NIHSS AFTER MECHANICAL THROMBECTOMY}

'B Maglinger*, , $\mathrm{M}$ Sands, ${ }^{1} \mathrm{~J}$ Frank, ${ }^{1} \mathrm{~A}$ Trout, ${ }^{2} \mathrm{~J}$ Roberts, ${ }^{2} \mathrm{~S}$ Grupke, ${ }^{1} \mathrm{~J}$ Turchan-Cholewo, ${ }^{1} \mathrm{~A}$ Stowe, ${ }^{2} \mathrm{~J}$ Fraser, ${ }^{1} \mathrm{~K}$ Pennypacker. 'Department of Neurology, University of Kentucky, Lexington, KY; ${ }^{2}$ Department of Neurosurgery, University of Kentucky, Lexington, $K Y$

\subsection{6/neurintsurg-2020-SNIS.278}

Introduction The University of Kentucky Blood and Clot Thrombectomy Registry and Collaboration (BACTRAC) protocol (clinicaltrials.gov NCT03153683) utilizes thrombectomy to isolate intracranial (i.e. distal to thrombus) arterial blood and systemic (i.e. carotid) arterial blood from thrombectomy procedures to better understand stroke. The objective of this study within that protocol was to investigate the relationship among Vascular Cell Adhesion Molecule 1 (VCAM1), hypertension (HTN), and stroke recovery in patients undergoing mechanical thrombectomy for emergent large vessel occlusion (ELVO) stroke. Here, we report how intracranial and systemic proteomic expression levels relate to HTN and the change in National Institute of Health Stroke Scale/Score (NIHSS) from admittance to discharge.

Methods Intracranial and systemic plasma samples from 25 subjects underwent cardiometabolic proteomic analysis at Olink Proteomics (Olink Proteomics, Boston, MA). VCAM1 expression levels were reported as a Normalized Proteomic eXpressoin (NPX) for each subject. Demographic data were also reported including HTN and both admission NIHSS and discharge NIHSS. Linear regression analysis was run on both intracranial and systemic VCAM1 expression levels against $\%$ change in NIHSS ((Admittance NIHSS - Discharge NIHSS)/ Admittance NIHSS)). Two-tailed t-tests were run assessing VCAM1 expression with hypertension (HTN) vs. no hypertension. Data analysis was performed using IBM SPSS Statistics.

Results Linear regression analysis demonstrated increased expression of intracranial VCAM1 significantly correlated with a smaller\% change in NIHSS $(p=0.001)$. Similarly, increased systemic VCAM1 expression was also found to have a significant relationship with smaller\% change in NIHSS $(p=0.005)$. Subjects with hypertension had significantly higher intracranial $(p=0.03)$ and systemic $(p=0.001)$ VCAM1 levels compared to those without HTN.
Discussion VCAM1 mediates leukocyte-endothelial cell adhesion and has been shown to play a role in atherosclerotic plaques, endothelial integrity and function, and stroke recurrence. This study takes a novel approach of sampling both intracranial and systemic arterial blood during an ELVO stroke. We found increased intracranial and systemic VCAM1 independently correlate with a smaller\% change in NIHSS. Although preliminary, these results may suggest an informative role of VCAM1 levels at the time of infarct. Additionally, within our cohort, those with HTN had higher levels of intracranial and systemic VCAM1. These data are in line with previous studies suggesting VCAM1 may be a marker of endothelial damage due to HTN. To better understand our findings, we plan to perform subset analyses investigating VCAM1 levels in relation to stroke recurrence, dyslipidemia, infarct time and infarct volume. As VCAM1 plays a role in leukocyte recruitment during the inflammatory state, we also plan to investigate how the antioxidant superoxide dismutase 1 (SOD1) may modulate intracranial and systemic levels of VCAM1 and influence functional recovery based on NIHSS.

Disclosures B. Maglinger: None. M. Sands: None. J. Frank: None. A. Trout: None. J. Roberts: None. S. Grupke: None. J. Turchan-Cholewo: None. A. Stowe: 4; C; Cerelux, LLC. J. Fraser: 2; C; Stream Biomedical, Medtronic, Penumbra. 4; C; Cerelux, LLC; Fawkes Biotechnology. K. Pennypacker: 4; C; Cerelux, LLC.

\section{LB-007 RESULTS OF HYBRID STUDY, PROSPECTIVE RANDOMIZED MULTICENTER STUDY OF HYDROGEL COIL VS BARE PLATINUM COIL FOR INTRACRANIAL ANEURYSMS}

${ }^{1} \mathrm{~N}$ Sakai ${ }^{*},{ }^{1} \mathrm{H}$ Imamura, ${ }^{1} \mathrm{C}$ Sakai, ${ }^{2} \mathrm{~A}$ Hyodo, ${ }^{3} \mathrm{Y}$ Ito, ${ }^{4} \mathrm{Y}$ Matsumaru, ${ }^{5} \mathrm{~S}$ Miyachi ${ }^{6} \mathrm{~S}$ Yoshimura, ${ }^{7} \mathrm{M}$ Sasaki, ${ }^{8} \mathrm{~K}$ Ogasawara, ${ }^{9} \mathrm{~S}$ Miyamoto, ${ }^{9} \mathrm{~S}$ Miyamoto, ${ }^{10} \mathrm{M}$ Ezura, ${ }^{11}$ I Nakahara, ${ }^{9} \mathrm{~A}$ Ishii, ${ }^{12} \mathrm{~T}$ Higashi. ${ }^{1}$ Neurosurgery, Kobe City Medical Center General Hospital, Kobe, Japan; ${ }^{2}$ Neurosurgery, Dokkyo Medical Univesity Saitama Medical Center, Koshigaya, Japan; ${ }^{3}$ Neurosurgery, Shinrakuen Hospital, Niigata, Japan; ${ }^{4}$ Neurosurgery, Tsukuba University, Tsukuba, Japan; ${ }^{5}$ Neurosurgery, Aichi Medical University, Nagakute, Japan; ${ }^{6}$ Neurosurgery, Hyogo College of Medicine, Nishinomiya, Japan; ${ }^{7}$ Radiology, Iwate Medical University, Morioka, Japan; ${ }^{8}$ Neurosurgery, Iwate Medical Unuversity, Morioka, Japan; ${ }^{9}$ Neurosurgery, Kyoto University, Kyoto, Japan; ${ }^{10}$ Neurosurgery, Sendai Medical Center, Sendai, Japan; "Neurosurgery, Fujita Health University, Toyoake, Japan; ${ }^{12}$ Neurosurgery, Fukuoka University Chikushi Hospital, Chikushino, Japan

\subsection{6/neurintsurg-2020-SNIS.279}

Purpose To present results of HYBRID study, whether second generation hydrogel coils (HGC) for the treatment of intracranial aneurysms improves outcomes compared with bare platinum coils.

Material and Methods HYBRID study is investigator-initiated, prospective, randomized, multicenter trial with open label treatment, blinded endpoint evaluation in Japan (NCT01516658). Inclusion criteria are 20-80 age, 7-20 mm size, unruptured or ruptured with WFNS grade I-III. For hydrogel coil arm ( $\mathrm{H}$ group), recommended to use HGC $50 \%$ length or more with maximum effort and for bare platinum coil arm (B group) prohibited to use any HGC and surface modificated coil. Primary endpoint is recanalization, evaluated on DSA at 1-year after embolization, and late aneurysm rupture, re-treatment and adverse events are secondary endpoints. 